\title{
Dissecting mRNA decay and translation inhibition during iron deficiency
}

Lucía Ramos-Alonsoํㅜ, Antonia María Romero ${ }^{1}$ Julio Polaina ${ }^{1}$, Sergi Puig1,* and María Teresa Martínez-Pastor ${ }^{2, *}$

${ }^{1}$ Departamento de Biotecnología, Instituto de Agroquímica y Tecnología de Alimentos (IATA), Consejo Superior de Investigaciones Científicas (CSIC), C/ Agustín Escardino 7, Paterna 46980, Valencia, Spain.

${ }^{2}$ Departamento de Bioquímica y Biología Molecular, Universitat de València, Ave. Doctor Moliner 50, Burjassot 46100, Valencia, Spain.

*Corresponding authors: Sergi Puig (spuig@iata.csic.es; +34 963900 022); María Teresa Martínez-Pastor (maria.teresa.martinez@uv.es; +34 963543 629).

ORCIDs: Lucía Ramos-Alonso: 0000-0002-1828-4115; Antonia María Romero: 0000-0002-2727-279X; Julio Polaina: 0000-0001-9912-0640; Sergi Puig: 0000-0002-1856-490X; María Teresa Martínez-Pastor: 0000-0002-4128-958X. 


\section{Abstract}

Iron participates as a vital cofactor in multiple metabolic pathways. Despite its abundance, iron bioavailability is highly restricted in aerobic and alkaline environments. Therefore, living organisms have evolved multiple adaptive mechanisms to respond to iron scarcity. These strategies include a global remodeling of iron metabolism directed to optimize iron utilization. In the baker's yeast Saccharomyces cerevisiae, this metabolic reorganization is accomplished to a large extent by an mRNA-binding protein called Cth2. Yeast Cth2 belongs to a conserved family of tandem zinc finger containing proteins that specifically bind to transcripts containing AU-rich elements and promote their turnover. A recent study has revealed that Cth2 also inhibits the translation of its target mRNAs. Interestingly, the mammalian Cth2 ortholog known as tristetraprolin (aka TTP/TIS11/ZFP36), which is also implicated in controlling iron metabolism, promotes the decay and prevents the translation of its regulated transcripts. These observations open the possibility to study the relative contribution of altering mRNA stability and translation to the physiological adaptation to iron deficiency, the function played by the different domains within the mRNA-binding protein, and the potential factors implicated in coordinating both post-transcriptional events.

Keywords: Protein translation, mRNA degradation, iron deficiency, yeast, tristetraprolin, Cth2. 


\section{Introduction}

Iron is an essential element for all eukaryotic organisms because it participates as a redox cofactor in many biological processes, including the generation of energy by oxidative phosphorylation and the biosynthesis of the major cellular components: proteins, lipids and DNA. When present in excess, iron participates in Fenton-type reactions that generate reactive oxygen species (ROS) that can damage lipids, proteins and nucleic acids (Meneghini 1997). The extremely low solubility of ferric iron at physiological conditions frequently leads in humans to the development of iron deficiency anemia, which is considered the most widespread nutritional disorder, with large impact on children and pregnant women (Baynes and Bothwell 1990, Sanvisens and Puig 2011). Moreover, iron imbalances lead to several human diseases including hereditary hemochromatosis, aceruloplasminemia and Friedreich's ataxia (Muckenthaler, et al. 2017).

Living organisms, ranging from bacteria to mammals, have evolved intricate regulatory networks to appropriately respond to iron starvation (Martins, et al. 2018, Muckenthaler, et al. 2017, Salvail and Masse 2012, Sanvisens and Puig 2011). In particular, the post-transcriptional regulation of gene expression plays a central role in controlling iron homeostasis. In several bacterial species, iron starvation activates the expression of the RyhB-like small noncoding RNAs, which degrade transcripts encoding proteins involved in iron metabolism and storage (Salvail and Masse 2012). Another family of small regulatory RNAs, called IsaR1 in the cyanobacterium Synechocystis, post-transcriptionally represses the photosynthetic electron transport chain in response to iron starvation by directly targeting certain 
mRNAs encoding components of the chain, maintaining the regulation of ironsulfur cluster biogenesis, and via the inhibition of the tetrapyrrole biosynthesis pathway (Carrier, et al. 2017, Georg, et al. 2017). In mammals, the iron regulatory proteins IRP1 and IRP2 bind to specific stem-loop RNA structures, known as iron responsive elements (IREs), located in the non-coding regions of a set of transcripts implicated in cellular iron homeostasis, to modulate its stability and translation efficiency (Muckenthaler, et al. 2017, Thompson and Bruick 2012). Finally, mammals express various proteins characterized by the presence of two tandem zinc fingers (TZFs) of the $\mathrm{Cx}_{8} \mathrm{Cx}_{5} \mathrm{Cx}_{3} \mathrm{H}$ type that specifically interact with AU-rich elements (AREs: 5'-UUAUUUAUU-3') present in the 3'-untranslated region (3'-UTR) of many mRNAs, promoting their degradation and inhibiting their translation (Carballo, et al. 1998). Human tristetraprolin (TTP/TIS11/ZFP36) is the most studied member of this TZF-containing family of RNA-binding proteins (Brooks and Blackshear 2013, Wells, et al. 2017). In addition to its antiinflammatory and tumor suppressor function (Clark and Dean 2016, Guo, et al. 2017, Patial and Blackshear 2016), TTP is highly expressed in response to iron deficiency (Bayeva, et al. 2012). Under these conditions, TTP controls the expression of mRNAs critical for mammalian iron metabolism, such as the transferrin receptor, and influences cell survival (Bayeva, et al. 2012). Recent data have demonstrated that TTP is also necessary for maintaining cardiac function during iron starvation by promoting the turnover of mRNAs of mitochondrial ironcontaining proteins, and thus avoiding electron leakage and oxidative damage (Sato, et al. 2018). 


\section{A central role for the Cth2 mRNA-binding protein in the response of yeast cells to iron deficiency.}

The baker's yeast Saccharomyces cerevisiae has developed sophisticated mechanisms to adapt to altered levels of iron (Kaplan and Kaplan 2009, Li and Ward 2018, Philpott and Protchenko 2008). Upon iron scarcity, yeast cells activate the transcription of a set of genes collectively known as the iron regulon, which encode the high-affinity iron uptake machinery at the plasma membrane, proteins implicated in the mobilization of iron from the vacuole and two mRNA-binding proteins known as Cth1 and Cth2 (Philpott and Protchenko 2008, Sanvisens and Puig 2011). Cth1 and Cth2 belong to the TTP-like family of mRNA-binding proteins (Puig, et al. 2005, Puig, et al. 2008, Thompson, et al. 1996, Vergara, et al. 2011). Genome-wide studies with yeast $\operatorname{cth} 2 \Delta$ cells cultivated in iron-depleted conditions and mRNA half-life experiments have shown that Cth2 promotes the destabilization of more than a hundred transcripts, which mainly encode irondependent proteins and proteins that participate in highly iron-consuming pathways (Puig, et al. 2005, Puig, et al. 2008). Specifically, Cth2-regulated processes include the mitochondrial electron transport chain, the tricarboxylic acid cycle, and key steps in the biosynthesis of various amino acids, lipids, deoxyribonucleoside triphosphates (dNTPs), heme, biotin and lipoic acid cofactors (Puig, et al. 2005, Puig, et al. 2008). Thus, Cth2 seems to optimize iron utilization during iron depletion by repressing dispensable iron-requiring pathways, such as mitochondrial respiration, and activating essential iron-dependent processes like the biosynthesis of dNTPs via ribonucleotide reductase (Sanvisens, et al. 2011, Sato, et al. 2018). The physiological relevance of Cth2 is highlighted by the 
significant growth defects associated to cells lacking a functional Cth2 protein when cultivated under iron-limiting conditions (Puig, et al. 2005). The function of Cth1, the second TTP-family yeast homolog, partially overlaps with Cth2. However, Cth1 performs a secondary role since cth1 $\Delta$ cells do not display a growth defect under iron starvation (Puig, et al. 2005, Puig, et al. 2008). The contribution of Cth1 to iron-deficiency is shown by the more severe growth defect of the $\operatorname{cth} 1 \Delta c t h 2 \Delta$ double mutant as compared to the $c t h 2 \Delta$ single mutant in these conditions (Puig, et al. 2005). Therefore, the experiments to address Cth2 function are usually performed in a cth1 $\Delta$ genetic background in order to exclude any interference of Cth1 (Puig, et al. 2005). CTH2 is also transiently activated in the response to oxidative stress (Castells-Roca, et al. 2016). In coordination to its Cth1 counterpart, Cth2 contributes to the resistance to ROS by decreasing the activity of mitochondrial respiration and by limiting the acquisition of oxidant-promoting iron through the reductive high-affinity uptake system (Castells-Roca, et al. 2016, Matsuo, et al. 2017, Sato, et al. 2018).

Although many changes in mRNA levels have been reported for yeast cells lacking CTH2, much less is known about its final effect on protein abundance. Previous studies have shown that Cth2 expression leads to a decrease in the protein levels of the succinate dehydrogenase subunits Sdh2 and Sdh4 (MartinezPastor M, et al. 2013, Ramos-Alonso, et al. 2018). By using specific antibodies, we have now addressed how Cth2 influences the levels of other iron-containing proteins when iron is scarce. Specifically, we have observed that the levels of ferrochelatase (Hem15), biotin synthase (Bio2), and to a minor extent aconitase (Aco1) increase in the absence of Cth2 (Figure 1). This result reinforces the notion 
that Cth2 limits the expression of multiple iron-dependent proteins when iron is scarce.

\section{Contribution of yeast Cth2 protein domains to mRNA decay and translational} inhibition.

The mechanism proposed for Cth2-mediated mRNA decay initiates with the cotranscriptional binding of Cth2 to its target mRNAs in the nucleus (MartinezPastor MT, et al. 2013, Prouteau, et al. 2008, Vergara, et al. 2011). Then, Cth2 either interferes with the polyadenylation machinery, leading to extended transcripts that are processed in the nucleus, or translocates to the cytoplasm where the mRNA is degraded from 5' to $3^{\prime}$ (Prouteau, et al. 2008, Vergara, et al. 2011). Binding to its target mRNAs is required for Cth2 export to the cytoplasm, since Cth2 proteins mutated in its TZFs, which do not associate to transcripts, are trapped in the nucleus and do not promote mRNA turnover (Vergara, et al. 2011).

The most conserved region in the TTP family of proteins is the TZF motif. Thus, the TZF of the fission yeast Schizosacchromyces pombe family member is functionally interchangeable with the analogous domain of other distantly related organisms including humans, insects, plants and pathogenic yeasts (Wells, et al. 2015). To date, the overall structure of TTP-type proteins has not been elucidated. However, the structure of the human TIS11d TZF domain associated to the single stranded RNA oligonucleotide 5'-UUAUUUAUU-3' has been solved, showing two highly similar $\mathrm{Cx}_{8} \mathrm{Cx}_{5} \mathrm{Cx}_{3} \mathrm{H}$ zinc fingers bound to separate 5'-UAUU-3' motifs (Hudson, et al. 2004). By using this sequence as a template, we have predicted now 
a potential structure for the yeast Cth2 TZF domain interacting with the mRNA (Figure 2). Beyond the TZF, the primary sequence conservation of this family of proteins decreases. Bioinformatic analyses of the mammalian and Drosophila melanogaster TIS11/TTP suggest that both proteins lack a stable tertiary structure, showing highly unstructured amino-terminal and carboxy-terminal regions (NTD and CTD) aside of the TZFs and short stretches at both ends of the protein (Ngoc, et al. 2014). These intrinsically disordered regions could facilitate the exposure of motifs implicated in the recruitment of other proteins. In fact, deletion of either the NTD or the CTD of TTP abrogates function (Rigby, et al. 2005). In addition to its TZFs, S. cerevisiae Cth2 protein contains three regions that are conserved among the genus Saccharomyces: CR1 and CR2, which localize to its NTD region, and CR3, which is present in its CTD end (Prouteau, et al. 2008). Similarly to other TTP family members, the bioinformatic analysis of the Cth2 NTD with the IUPred2A algorithm (Meszaros, et al. 2018) suggests that the NTD of yeast Cth2 protein acquires an unstructured conformation. Interestingly, in the structural model of Cth2 CTD, CR3 folds back into the TZFs (Figure 2). This is in agreement with the lack of functionality of any CTD-tagged Cth2 protein, as the tag likely interferes with the TZFs function (Prouteau, et al. 2008, Puig, et al. 2005, Vergara, et al. 2011). None of these three conserved domains (CR1, CR2 and CR3) is necessary for Cth2 nucleocytoplasmic shuttling and for binding to ARE-containing mRNAs, which depend on the integrity of the TZFs (Prouteau, et al. 2008, Ramos-Alonso, et al. 2018, Vergara, et al. 2011). Importantly, the deletion of CR1 alone, or in combination with CR2, leads to an increase in the steady-state levels of Cth2 mRNA targets and to the appearance of new extended transcript species during iron depletion; whereas no changes are observed for a Cth2 protein lacking CR3 
(Prouteau, et al. 2008, Ramos-Alonso, et al. 2018). These results suggest that Cth2 NTD is implicated in mRNA decay and transcription termination by probably recruiting the mRNA decay and termination machineries, whereas the CTD does not participate in these processes. Consistent with this, recent mRNA half-life measurements have demonstrated that the CR3 is dispensable for the turnover of SDH4 mRNA in response to iron-depleted conditions (Ramos-Alonso, et al. 2018). By using protein/mRNA ratios and polysome profiles, we have shown that, in addition to promoting mRNA decay, Cth2 inhibits the translation of its target mRNAs in a process that depends on the integrity of the ARE within the transcript and of Cth2 TZFs (Ramos-Alonso, et al. 2018). Remarkably, we have observed that not only the NTD but also Cth2 CTD contribute to repress the translation of the target mRNAs (Ramos-Alonso, et al. 2018). The fact that CR3 is only necessary to inhibit mRNA translation, but does not alter the stability of the transcript, opened the possibility to study the contribution of translation repression to cell growth and to the regulatory function played by Cth2 during iron starvation. Cells expressing a Cth2 protein lacking the CR3 (Cth2 $\Delta$ C52) displayed a growth defect under iron-depleted conditions, emphasizing the importance of Cth2 CTD (RamosAlonso, et al. 2018). The role played by the Cth2-mediated regulation of mRNA translation is stressed by the striking increase in the protein levels of various ironusing enzymes displayed in Cth2 $\Delta$ C52-expressing cells (Figure 1). Altogether these data strongly suggest a central contribution of Cth2 CTD to the adaptation of yeast cells to iron depletion via translation repression.

\section{Mechanism for Cth2-mediated repression of protein translation}


Eukaryotic mRNA decay and protein translation are closely related processes. In general, mRNA turnover occurs as a consequence of translational repression (Huch and Nissan 2014). For instance, the yeast decapping activators Dhh1 and Pat1 promote decapping and mRNA turnover after inhibiting translation (Parker 2012, Roy and Jacobson 2013). However, this intimate interconnection is far from being straightforward. There are unstable mRNAs that are translated efficiently, while other highly stable transcripts remain untranslated (Roy and Jacobson 2013). In fact, degradation of actively translated mRNAs occurs in some instances (Hu, et al. 2009). Polysome loading is even a prerequisite to the mRNA decay exerted by some RNases (Mino, et al. 2015). There are also examples in which both processes have been separated, such as the mouse TTP homolog Zfp3611, which represses the translation the $V E G F$ mRNA without altering its mRNA stability (Bell, et al. 2006).

After establishing that Cth2 plays an important role in regulating the translation of many iron-containing proteins under iron deficiency, several questions arise. We do not know yet if the Cth2 translational repression function comes first or after its role as an activator of mRNA decay. Our studies with truncated Cth2 proteins suggest that Cth2 CTD could preferentially interact with factors that control translation, while proteins implicated in both mRNA turnover and translation would associate to its NTD (Ramos-Alonso, et al. 2018). Many conserved mRNA decay and translational repression regulatory factors interact with TTP, the mammalian Cth2 ortholog, including the RNA helicase RCK/p54, the CCR4-NOT complex, the cap-binding factor eIF4E2 or the Poly-A-Binding Protein (Fu, et al. 2016, Lykke-Andersen and Wagner 2005, Qi, et al. 2012, Tao and Gao 2015, Zhang, et al. 2017). The identification of the machinery that allows Cth2 to 
post-transcriptionally regulate gene expression could contribute to understand the connections between mRNA decay and translation. Dhh1, the yeast RCK/p54 homolog that functions as an activator of decapping and a repressor of translation under stress conditions, is a plausible candidate to mediate Cth2 translational repression for several reasons. First, we observed a genetic interaction between CTH2 and DHH1 genes, since the deletion of DHH1 rescues the growth defect of Cth2-overexpressing cells. Secondly, the Cth2 target mRNA SDH4 stabilizes in irondeficient $d h h 1 \Delta$ cells. And thirdly, yeast two hybrid experiments indicate that Cth2 interacts with the CTD of Dhh1 (Pedro-Segura, et al. 2008). However, the domains of Cth2 involved in this interaction have not yet been determined. Dhh1 has been shown to act as a general translational repressor when overexpressed (Coller and Parker 2005). Although initially thought to act at the level of translation initiation, it has been recently postulated that Dhh1 slows down the movement of ribosomes on the mRNAs and, as a consequence, promotes decapping and decay (Sweet, et al. 2012). Therefore, the repression of protein translation during the elongation phase would occur prior to the activation of decapping and independently of it (Carroll, et al. 2011, Sweet, et al. 2012). Nonetheless, many details about the molecular mechanism of Dhh1-mediated repression of translation are still unknown, and an additional role of Dhh1 at the initiation step cannot yet be excluded. More information on the mode of action of Dhh1 as well as on the domains of Cth2 involved in the interaction with Dhh1 would be necessary to fully understand the particular role of both proteins in ARE-mediated translational inhibition and mRNA decay under iron deficiency. 


\section{Conclusions and perspectives}

The post-transcriptional regulation of gene expression plays a central role in iron homeostasis, and the baker's yeast is not an exception. The RNA-binding protein Cth2 modulates iron metabolism by controlling both the stability and the translation of ARE-containing mRNAs during the adaptation to iron starvation (Puig, et al. 2005, Ramos-Alonso, et al. 2018). However, the relative contribution and the connections between Cth2-dependent mRNA turnover and translation have not been fully elucidated. Further studies on the sequential assembly of regulatory proteins onto the different Cth2 domains and onto the target mRNA could improve our current understanding of the mechanisms that posttranscriptionally regulate gene expression. We have previously shown that the

conserved RNA helicase Dhh1 participates in the Cth2-mediated degradation of specific mRNAs under iron-deficient conditions (Pedro-Segura, et al. 2008). Since Dhh1 as a global regulator of mRNA translation, its involvement in controlling mRNA translation via Cth2 in response to low iron is a likely hypothesis that requires a thorough examination. 


\section{Materials and Methods}

Yeast strains, plasmids and growth conditions. To determine protein

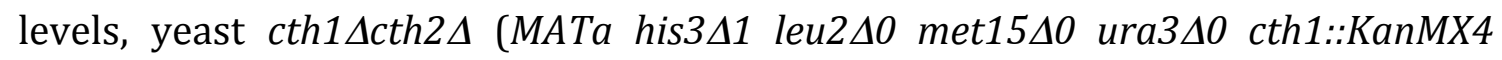
cth2::HisMX6) cells transformed with pRS416-GFP-CTH2, pRS416 or pRS416-GFPCTH2 2 C52 plasmids were cultivated for 6 hours in synthetic complete medium without uracil (SC-ura) supplemented with $100 \mu \mathrm{M}$ of the $\mathrm{Fe}^{2+}$-specific chelator bathophenanthroline disulfonate (BPS) (Puig, et al. 2005, Ramos-Alonso, et al. 2018, Sikorski and Hieter 1989, Vergara, et al. 2011).

Protein analyses. Total protein extracts were obtained by using the alkali method (Kushnirov 2000). Equal amounts of protein were resolved in SDS-PAGE gels and transferred to nitrocellulose membranes. Ponceau staining was used to assess protein transfer. Aconitase (Aco1p), biotin synthase (Bio2p) and ferrochelatase (Hem15p) were detected using specific antibodies from rabbit kindly provided by Dr. Roland Lill (Philipps-Universität Marburg, Germany). We used HRP-labeled secondary anti-rabbit antibody from donkey (Santa Cruz Biotech) and ECL Select Western blotting detection kit (GE Healthcare Life Sciences). Immunoblot images were obtained in an ImageQuant LAS 4000 mini Biomolecular Imager (GE Healthcare Life Sciences) and specific signals were quantified with ImageQuant TL analysis software (GE Healthcare Life Sciences). Aco1, Bio2 and Hem15 protein levels were quantified, and the relative average and standard deviation of three independent biological samples are indicated. Tailed tstudent tests were applied to evaluate statistical significance. 
Bioinformatic analyses. The structural model for the TZFs and the CTD of the yeast Cth2 protein (from amino acid 160 to 285) has been obtained by using the I-TASSER server for protein structure and function prediction (Yang, et al. 2015). PDB structures 1RGO (Hudson, et al. 2004) and 2CQE, which corresponds to the zinc-finger domain of human protein KIA1046, were used as templates. The IUPred2A software (iupred2a.elte.hu) was used to predict potential intrinsically unstructured protein regions (Meszaros, et al. 2018). 


\section{Acknowledgements}

We are grateful to Drs. Roland Lill and Ulrich Mühlenhoff for the antibodies used in this work and to Dr. Paula Alepuz for scientific discussions. This work was supported by predoctoral contracts from the Spanish Ministry of Economy, Industry and Competitiveness (MINECO) to L. R-A. and A. M. R.; grants BIO201787828-C2-1-P and BFU2015-71978-REDT from MINECO and "Fondo Europeo de Desarrollo Regional" (FEDER) funds to S. P.; and grant AICO/2015/004 from the “Generalitat Valenciana” to M. T. M.-P. 


\section{Figure legends}

Figure 1. The deletion of the Cth2 carboxy-terminal conserved region CR3 alters the levels of various iron-dependent proteins. Yeast cells expressing CTH2, empty vector or $C T H 2 \triangle C 52$ were grown in iron-deficient conditions to exponential phase. Total proteins were extracted and the levels of aconitase (Aco1p), biotin synthase (Bio2p) and ferrochelatase (Hem15p) determined. The average and standard deviation of three independent biological samples is shown. The asterisk $(*)$ indicates a significant p-value $(\leq 0.05)$ compared to CTH2expressing cells.

Figure 2. Structural model for the carboxy-terminal region of the yeast Cth2 protein. A model for the TZFs and the CTD of the yeast Cth2 protein (from amino acid 160 to 285) bound to single stranded RNA is represented. The RNA is shown in red, whereas the protein backbone is indicated in gray except for the CR3 (from amino acid 263 to 285), which is depicted in green. The histidine and cysteine residues that coordinate the two $\mathrm{Zn}^{2+}$ ions (symbolized by dark spheres) are displayed in blue and yellow color, respectively. TZF1: TZF on the NTD side; TZF2: TZF on the CTD side. 


\section{References}

Bayeva M, Khechaduri A, Puig S, Chang HC, Patial S, Blackshear PJ, Ardehali H (2012) mTOR regulates cellular iron homeostasis through tristetraprolin. Cell Metab 16: 645-657 doi: 10.1016/j.cmet.2012.10.001

Baynes RD, Bothwell TH (1990) Iron deficiency. Annu Rev Nutr 10: 133-148 doi: 10.1146/annurev.nu.10.070190.001025

Bell SE, Sanchez MJ, Spasic-Boskovic O, Santalucia T, Gambardella L, Burton GJ, Murphy JJ, Norton JD, Clark AR, Turner M (2006) The RNA binding protein Zfp36l1 is required for normal vascularisation and post-transcriptionally regulates VEGF expression. Dev Dyn 235: 3144-3155 doi: 10.1002/dvdy.20949

Brooks SA, Blackshear PJ (2013) Tristetraprolin (TTP): interactions with mRNA and proteins, and current thoughts on mechanisms of action. Biochim Biophys Acta 1829: 666-679 doi: 10.1016/j.bbagrm.2013.02.003

Carballo E, Lai WS, Blackshear PJ (1998) Feedback inhibition of macrophage tumor necrosis factor-alpha production by tristetraprolin. Science 281: 1001-1005 doi:

Carrier MC, Bourassa JS, Masse E (2017) Cellular Homeostasis: A Small RNA at the Crossroads of Iron and Photosynthesis. Curr Biol 27: R380-R383 doi: 10.1016/j.cub.2017.04.003

Carroll JS, Munchel SE, Weis K (2011) The DExD/H box ATPase Dhh1 functions in translational repression, mRNA decay, and processing body dynamics. J Cell Biol 194: 527-537 doi: 10.1083/jcb.201007151

Castells-Roca L, Pijuan J, Ferrezuelo F, Belli G, Herrero E (2016) Cth2 Protein Mediates Early Adaptation of Yeast Cells to Oxidative Stress Conditions. PLoS One 11: e0148204 doi: 10.1371/journal.pone.0148204

Clark AR, Dean JL (2016) The control of inflammation via the phosphorylation and dephosphorylation of tristetraprolin: a tale of two phosphatases. Biochem Soc Trans 44: 1321-1337 doi: 10.1042/BST20160166

Coller J, Parker R (2005) General translational repression by activators of mRNA decapping. Cell 122: 875-886 doi: 10.1016/j.cell.2005.07.012

Fu R, Olsen MT, Webb K, Bennett EJ, Lykke-Andersen J (2016) Recruitment of the 4EHP-GYF2 cap-binding complex to tetraproline motifs of tristetraprolin promotes repression and degradation of mRNAs with AU-rich elements. RNA 22: 373-382 doi: 10.1261/rna.054833.115

Georg J, Kostova G, Vuorijoki L, Schon V, Kadowaki T, Huokko T, Baumgartner D, Muller M, Klahn S, Allahverdiyeva Y, Hihara Y, Futschik ME, Aro EM, Hess WR (2017) Acclimation of Oxygenic Photosynthesis to Iron Starvation Is Controlled by the sRNA IsaR1. Curr Biol 27: 1425-1436 e1427 doi: 10.1016/j.cub.2017.04.010

Guo J, Qu H, Chen Y, Xia J (2017) The role of RNA-binding protein tristetraprolin in cancer and immunity. Med Oncol 34: 196 doi: 10.1007/s12032-017-1055-6

Hu W, Sweet TJ, Chamnongpol S, Baker KE, Coller J (2009) Co-translational mRNA decay in Saccharomyces cerevisiae. Nature 461: 225-229 doi: $10.1038 /$ nature08265 
Huch S, Nissan T (2014) Interrelations between translation and general mRNA degradation in yeast. Wiley Interdiscip Rev RNA 5: 747-763 doi: 10.1002/wrna.1244

Hudson BP, Martinez-Yamout MA, Dyson HJ, Wright PE (2004) Recognition of the mRNA AU-rich element by the zinc finger domain of TIS11d. Nat Struct Mol Biol 11: 257-264 doi: 10.1038/nsmb738

Kaplan CD, Kaplan J (2009) Iron acquisition and transcriptional regulation. Chem Rev 109: 4536-4552 doi: 10.1021/cr9001676

Kushnirov VV (2000) Rapid and reliable protein extraction from yeast. Yeast 16: 857-860 doi: 10.1002/1097-0061(20000630)16:9<857::AIDYEA561>3.0.CO;2-B

Li L, Ward DM (2018) Iron toxicity in yeast: transcriptional regulation of the vacuolar iron importer Ccc1. Curr Genet 64: 413-416 doi: 10.1007/s00294017-0767-7

Lykke-Andersen J, Wagner E (2005) Recruitment and activation of mRNA decay enzymes by two ARE-mediated decay activation domains in the proteins TTP and BRF-1. Genes Dev 19: 351-361 doi: 10.1101/gad.1282305

Martinez-Pastor M, Vergara SV, Puig S, Thiele DJ (2013) Negative feedback regulation of the yeast $\mathrm{CTH} 1$ and $\mathrm{CTH} 2$ mRNA binding proteins is required for adaptation to iron deficiency and iron supplementation. Mol Cell Biol 33: 2178-2187 doi: 10.1128/MCB.01458-12

Martinez-Pastor MT, de Llanos R, Romero AM, Puig S (2013) Post-transcriptional regulation of iron homeostasis in Saccharomyces cerevisiae. Int J Mol Sci 14: 15785-15809 doi: 10.3390/ijms140815785

Martins TS, Costa V, Pereira C (2018) Signaling pathways governing iron homeostasis in budding yeast. Mol Microbiol 10.1111/mmi.14009

Matsuo R, Mizobuchi S, Nakashima M, Miki K, Ayusawa D, Fujii M (2017) Central roles of iron in the regulation of oxidative stress in the yeast Saccharomyces cerevisiae. Curr Genet 63: 895-907 doi: 10.1007/s00294-017-0689-4

Meneghini R (1997) Iron homeostasis, oxidative stress, and DNA damage. Free Radic Biol Med 23: 783-792 doi:

Meszaros B, Erdos G, Dosztanyi Z (2018) IUPred2A: context-dependent prediction of protein disorder as a function of redox state and protein binding. Nucleic Acids Res 46: W329-W337 doi: 10.1093/nar/gky384

Mino T, Murakawa Y, Fukao A, Vandenbon A, Wessels HH, Ori D, Uehata T, Tartey S, Akira S, Suzuki Y, Vinuesa CG, Ohler U, Standley DM, Landthaler M, Fujiwara T, Takeuchi $O$ (2015) Regnase-1 and Roquin Regulate a Common Element in Inflammatory mRNAs by Spatiotemporally Distinct Mechanisms. Cell 161: 1058-1073 doi: 10.1016/j.cell.2015.04.029

Muckenthaler MU, Rivella S, Hentze MW, Galy B (2017) A Red Carpet for Iron Metabolism. Cell 168: 344-361 doi: 10.1016/j.cell.2016.12.034

Ngoc LV, Wauquier C, Soin R, Bousbata S, Twyffels L, Kruys V, Gueydan C (2014) Rapid proteasomal degradation of posttranscriptional regulators of the TIS11/tristetraprolin family is induced by an intrinsically unstructured region independently of ubiquitination. Mol Cell Biol 34: 4315-4328 doi: 10.1128/MCB.00643-14

Parker R (2012) RNA degradation in Saccharomyces cerevisae. Genetics 191: 671702 doi: 10.1534/genetics.111.137265 
Patial S, Blackshear PJ (2016) Tristetraprolin as a Therapeutic Target in Inflammatory Disease. Trends Pharmacol Sci 37: 811-821 doi: 10.1016/j.tips.2016.07.002

Pedro-Segura E, Vergara SV, Rodriguez-Navarro S, Parker R, Thiele DJ, Puig S (2008) The Cth2 ARE-binding protein recruits the Dhh1 helicase to promote the decay of succinate dehydrogenase SDH4 mRNA in response to iron deficiency. J Biol Chem 283: 28527-28535 doi: 10.1074/jbc.M804910200

Philpott CC, Protchenko 0 (2008) Response to iron deprivation in Saccharomyces cerevisiae. Eukaryot Cell 7: 20-27 doi: 10.1128/EC.00354-07

Prouteau M, Daugeron MC, Seraphin B (2008) Regulation of ARE transcript 3' end processing by the yeast Cth2 mRNA decay factor. EMBO J 27: 2966-2976 doi: $10.1038 /$ emboj.2008.212

Puig S, Askeland E, Thiele DJ (2005) Coordinated remodeling of cellular metabolism during iron deficiency through targeted mRNA degradation. Cell 120: 99-110 doi: 10.1016/j.cell.2004.11.032

Puig S, Vergara SV, Thiele DJ (2008) Cooperation of two mRNA-binding proteins drives metabolic adaptation to iron deficiency. Cell Metab 7: 555-564 doi: 10.1016/j.cmet.2008.04.010

Qi MY, Wang ZZ, Zhang Z, Shao Q, Zeng A, Li XQ, Li WQ, Wang C, Tian FJ, Li Q, Zou J, Qin YW, Brewer G, Huang S, Jing Q (2012) AU-rich-element-dependent translation repression requires the cooperation of tristetraprolin and RCK/P54. Mol Cell Biol 32: 913-928 doi: 10.1128/MCB.05340-11

Ramos-Alonso L, Romero AM, Soler MA, Perea-Garcia A, Alepuz P, Puig S, MartinezPastor MT (2018) Yeast Cth2 protein represses the translation of AREcontaining mRNAs in response to iron deficiency. PLoS Genet 14: e1007476 doi: 10.1371/journal.pgen.1007476

Rigby WF, Roy K, Collins J, Rigby S, Connolly JE, Bloch DB, Brooks SA (2005) Structure/function analysis of tristetraprolin (TTP): p38 stress-activated protein kinase and lipopolysaccharide stimulation do not alter TTP function. J Immunol 174: 7883-7893 doi:

Roy B, Jacobson A (2013) The intimate relationships of mRNA decay and translation. Trends Genet 29: 691-699 doi: 10.1016/j.tig.2013.09.002

Salvail H, Masse E (2012) Regulating iron storage and metabolism with RNA: an overview of posttranscriptional controls of intracellular iron homeostasis. Wiley Interdiscip Rev RNA 3: 26-36 doi: 10.1002/wrna.102

Sanvisens N, Bano MC, Huang M, Puig S (2011) Regulation of ribonucleotide reductase in response to iron deficiency. Mol Cell 44: $759-769$ doi: 10.1016/j.molcel.2011.09.021

Sanvisens N, Puig S (2011) Causes and consequences of nutritional iron deficiency in living organisms. In: Merkin TC (ed) Biology of starvation in humans and other organisms. Nova Science Publishers, pp. 245-276.

Sato T, Chang HC, Bayeva M, Shapiro JS, Ramos-Alonso L, Kouzu H, Jiang X, Liu T, Yar S, Sawicki KT, Chen C, Martinez-Pastor MT, Stumpo DJ, Schumacker PT, Blackshear PJ, Ben-Sahra I, Puig S, Ardehali H (2018) mRNA-binding protein tristetraprolin is essential for cardiac response to iron deficiency by regulating mitochondrial function. Proc Natl Acad Sci U S A 115: E6291E6300 doi: 10.1073/pnas.1804701115 
Sikorski RS, Hieter P (1989) A system of shuttle vectors and yeast host strains designed for efficient manipulation of DNA in Saccharomyces cerevisiae. Genetics 122: 19-27 doi:

Sweet T, Kovalak C, Coller J (2012) The DEAD-box protein Dhh1 promotes decapping by slowing ribosome movement. PLoS Biol 10: e1001342 doi: 10.1371/journal.pbio.1001342

Tao X, Gao G (2015) Tristetraprolin Recruits Eukaryotic Initiation Factor 4E2 To Repress Translation of AU-Rich Element-Containing mRNAs. Mol Cell Biol 35: 3921-3932 doi: 10.1128/MCB.00845-15

Thompson JW, Bruick RK (2012) Protein degradation and iron homeostasis. Biochim Biophys Acta 1823: 1484-1490 doi: 10.1016/j.bbamcr.2012.02.003

Thompson MJ, Lai WS, Taylor GA, Blackshear PJ (1996) Cloning and characterization of two yeast genes encoding members of the CCCH class of zinc finger proteins: zinc finger-mediated impairment of cell growth. Gene 174: 225-233 doi:

Vergara SV, Puig S, Thiele DJ (2011) Early recruitment of AU-rich elementcontaining mRNAs determines their cytosolic fate during iron deficiency. Mol Cell Biol 31: 417-429 doi: 10.1128/MCB.00754-10

Wells ML, Hicks SN, Perera L, Blackshear PJ (2015) Functional equivalence of an evolutionarily conserved RNA binding module. J Biol Chem 290: 2441324423 doi: 10.1074/jbc.M115.673012

Wells ML, Perera L, Blackshear PJ (2017) An Ancient Family of RNA-Binding Proteins: Still Important! Trends Biochem Sci 42: 285-296 doi: 10.1016/j.tibs.2016.12.003

Yang J, Yan R, Roy A, Xu D, Poisson J, Zhang Y (2015) The I-TASSER Suite: protein structure and function prediction. Nat Methods 12: 7-8 doi: $10.1038 /$ nmeth.3213

Zhang X, Chen X, Liu Q, Zhang S, Hu W (2017) Translation repression via modulation of the cytoplasmic poly(A)-binding protein in the inflammatory response. Elife 610.7554/eLife.27786 


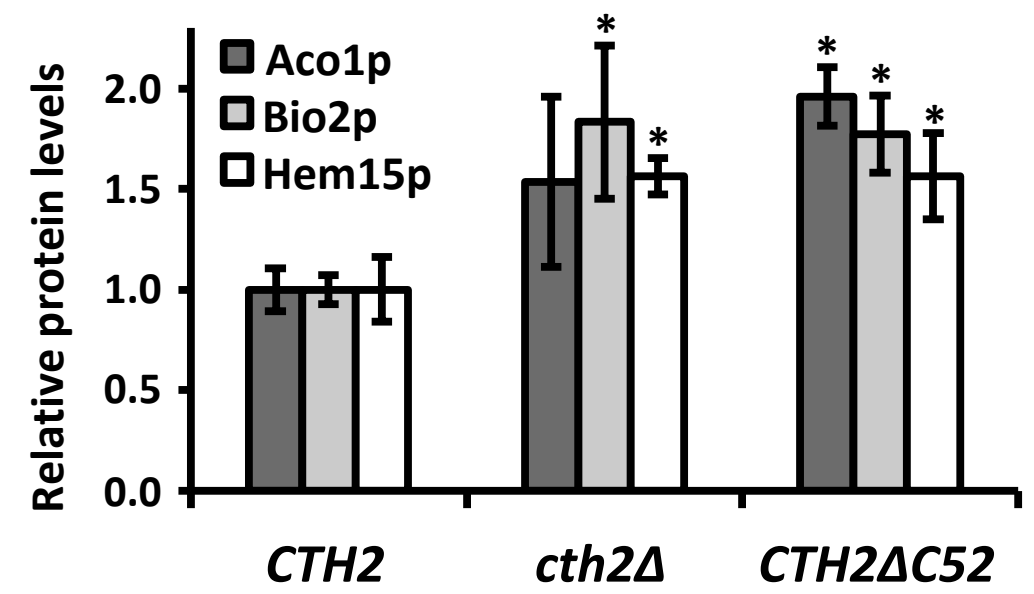

Figure 1 


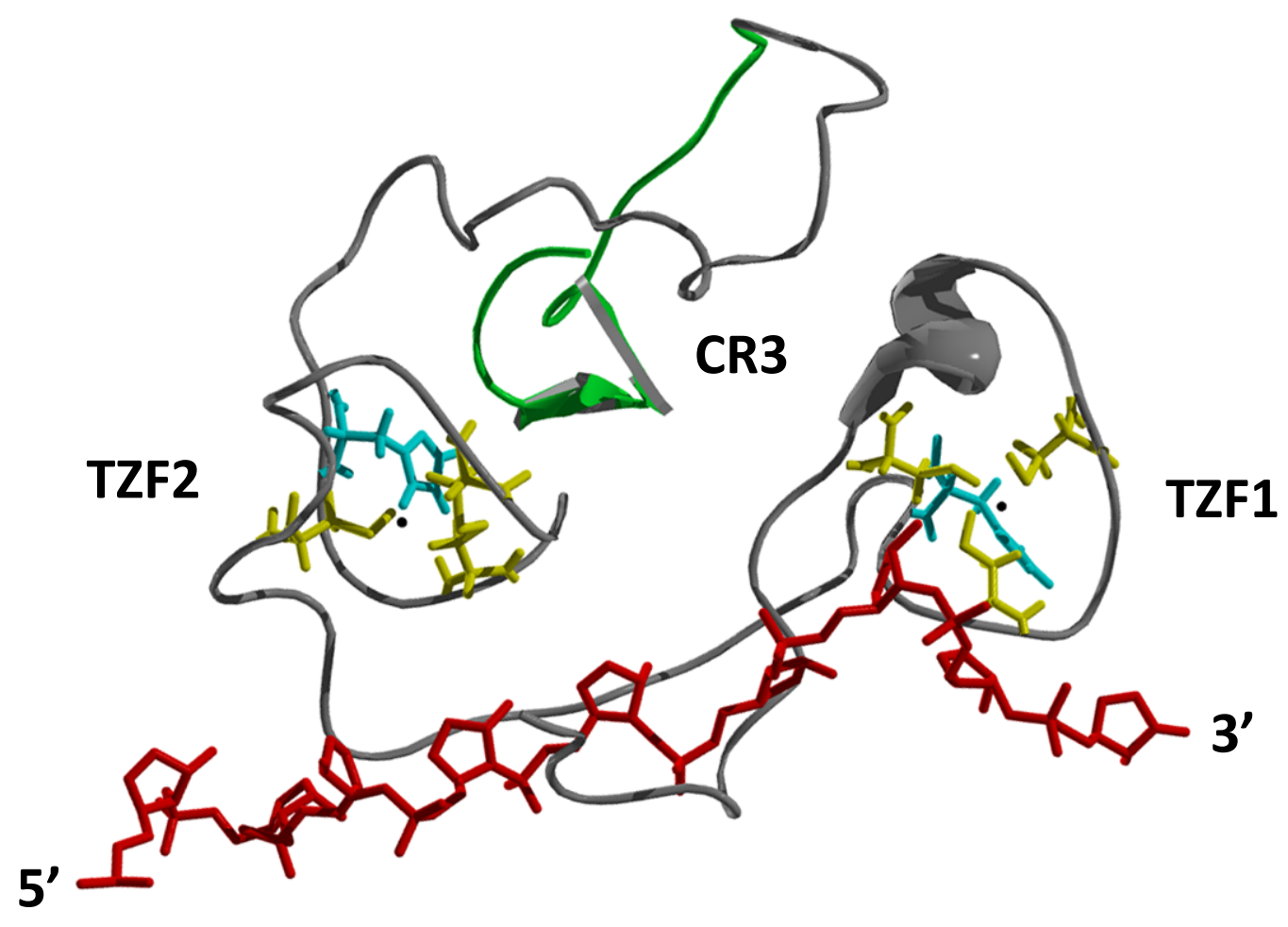

Figure 2 